\title{
Demethylation by low-dose 5-aza-2'- deoxycytidine impairs 3D melanoma invasion partially through miR-199a-3p expression revealing the role of this $\mathrm{miR}$ in melanoma
}

\author{
Cécile Desjobert $^{1 \dagger}$, Arnaud Carrier $^{1 \dagger}$, Audrey Delmas $^{2}$, Diego M. Marzese ${ }^{3}$, Antoine Daunay $^{4}$, Florence Busato ${ }^{5}$, \\ Arnaud Pillon ${ }^{6}$, Jörg Tost ${ }^{5}$, Joëlle Riond ${ }^{1,7}$, Gilles Favre ${ }^{2}$, Chantal Etievant ${ }^{9}$ and Paola B. Arimondo ${ }^{1,8^{*}}$ (D)
}

\begin{abstract}
Background: Efficient treatments against metastatic melanoma dissemination are still lacking. Here, we report that low-cytotoxic concentrations of 5-aza-2'-deoxycytidine, a DNA demethylating agent, prevent in vitro 3D invasiveness of metastatic melanoma cells and reduce lung metastasis formation in vivo.

Results: We unravelled that this beneficial effect is in part due to MIR-199A2 re-expression by promoter demethylation. Alone, this miR showed an anti-invasive and anti-metastatic effect. Throughout integration of micro-RNA target prediction databases with transcriptomic analysis after 5-aza-2'-deoxycytidine treatments, we found that miR-199a-3p downregulates set of genes significantly involved in invasion/migration processes. In addition, analysis of data from melanoma patients showed a stage- and tissue type-dependent modulation of MIR-199A2 expression by DNA methylation.
\end{abstract}

Conclusions: Thus, our data suggest that epigenetic- and/or miR-based therapeutic strategies can be relevant to limit metastatic dissemination of melanoma.

Keywords: DNA methylation, microRNA, Melanoma aggressiveness, Epigenetics

\section{Background}

Cutaneous metastatic melanoma is the deadliest form of skin cancer, with a 5-year survival rate particularly low $(<$ $10 \%)$ and increasing incidence [1]. Until recently, patients with metastatic melanoma had few treatment options, including surgery, radiation therapy and/or chemotherapy, giving transient and limited results. Since 2011, new targeted therapies against mutated BRAF (vemurafenib) and immunotherapies such as anti-CTLA4 (ipilimumab) and anti-PD1/PD-L1 antibodies emerged, giving very promising long-term responses. Despite the efficacy of these treatments in clinical trials, resistance rapidly arises after

\footnotetext{
* Correspondence: paola.arimondo@pasteur.fr

${ }^{\dagger}$ Cécile Desjobert and Arnaud Carrier contributed equally to this work.

${ }^{1}$ FRE no. 3600 CNRS, Epigenetic Targeting of Cancer (ETaC), Toulouse, France ${ }^{8}$ Institut Pasteur CNRS UMR3523, Epigenetic Chemical Biology, Paris, France Full list of author information is available at the end of the article
}

vemurafenib and immunotherapies can induce severe side effects limiting the therapeutic response [2]. In addition, not all patients respond to these therapies and no molecular marker is available to predict patient responsiveness to immunomodulators. Therefore, there is still a strong need for novel strategies.

Interestingly, targeting of the epigenetic regulation is promising for the development of new anticancer treatments in solid tumours [3]. In the past few years, various studies reported on epigenetic alterations in metastatic melanoma, more specifically DNA methylation, and associated them with disease progression [4-9]. DNA methylation, catalysed by DNA methyltransferases (DNMT), is a well-studied epigenetic change in cancer. In the last decade, several epigenetic drugs, such as DNA methylation inhibitors (DNMTi), azacitidine (5aza, Vidaza ${ }^{\circ}$ ) and decitabine $\left(5 \operatorname{azadC}, \operatorname{Dacogen}^{\mathrm{Tw}}\right)$, have been approved for the 
treatment of haematological tumours. In solid tumours, the use of epigenetic therapies is breaking through [10]. Hypomethylation of repetitive DNA elements and hypermethylation of $\mathrm{CpG}$ islands located in promoter regions of specific genes are hallmarks of melanoma malignancy [10-13]. Specific methylation signatures were also found for melanoma tumours harbouring BRAF mutations $[8$, 14]. Thus, the use of an epigenetic strategy, combined with relevant $\mathrm{CpG}$ island methylator phenotype (CIMP) [15] as markers for the prediction of prognosis or therapeutic response, could be envisioned for metastatic melanoma treatment $[10,16,17]$.

In this context, we investigated the implication of DNA methylation in the aggressiveness of metastatic melanoma and whether the use of demethylating agents can reverse its invasive phenotype. We focused our attention on microRNAs (miRs), since these endogenous non-coding RNAs of 19-25 nt can modulate the expression of several target genes via translational blockade and/or transcript degradation and are broadly involved in cancer progression. Furthermore, some miRs are described to be epigenetically regulated $[12,18]$ and to participate in the acquisition of invasive capabilities of cancer cells, including melanoma [19-21]. Here, we identified one specific hypermethylated miR gene, MIR199A2, whose expression restoration by low doses of 5-aza-2'-deoxycytidine or miR transfection impacts on in vitro cell invasiveness and in vivo metastatic formation.

\section{Results}

5azadC impairs melanoma cell invasiveness in a 3D invasion model at low non-cytotoxic doses

To date, a limited number of studies have been published depicting the effects of DNMTi on metastatic melanoma aggressiveness and most of them used 2D culture conditions to study invasiveness [22-24], at the exception of the study in combination with IFN-1 [25]. We set up a spheroid invasion assay to explore 3D invasive capacities of metastatic melanoma cells, since this model better preserves cellular features such as cell morphology and cell-cell and cell-extracellular matrix adhesion, known to be important for metastatic dissemination [26]. The metastatic melanoma WM-266-4 cell line constitutively expressing GFP (called herein WM-266-4 GFP) was used to follow the 3D cell invasion. Non-treated and 5azadC-treated WM-266-4-GFP cells were plated to form spheroids, which were embedded in collagen and monitored $24 \mathrm{~h}$ afterwards by fluorescent microscopy (Fig. 1a). Prior to measuring its effect on 3D cell invasion, the cell sensitivity to daily treatments of 5azadC, the reference DNMTi, was tested during 3 days. The WM-266-4 GFP cell line displayed a similar viability dose-response curve to the parental cell line WM-266-4, with an EC50 of $100 \mathrm{nM}$ at 7 days (Additional file 1: Figure S2A). In addition, cell sensitivity to 5 azadC resulted unchanged under different culture conditions, as shown by comparing $2 \mathrm{D}$ and $3 \mathrm{D}$ cultured cells (Additional file 1: Figure S2B).

Hence, low concentrations of $5 \mathrm{azadC}$, inducing little cell death and inhibition of cell proliferation $(\mathrm{EC} 50=100 \mathrm{nM})$, were chosen for the $3 \mathrm{D}$ invasion assay to limit non-specific cytotoxic effects and favour an epigenetic effect. As illustrated in Fig. 1b, c, 5azadC induced a dose-dependent inhibition of 3D cell invasion, with a significant decrease of the invasion index starting at $3.2 \mathrm{nM}$ $(P$ value $<0.001)$. The anti-metabolic compound cytarabine (araC), structurally similar to 5azadC and commonly used in chemotherapies [27], was used as control at concentrations resulting in $<10 \%$ of cell death $(1 \mathrm{nM}$, Additional file 1: Figure S3A). Unlike 5azadC, at the equi-cytotoxic concentration, araC did not cause cell invasion inhibition (Additional file 1: Figure S3B and S3C), confirming a different mechanism of action for 5azadC.

Next, the DNA demethylating activity of the drug was determined by following the global DNA methylation level in spheroids recovered before inclusion in collagen (at day 7, Fig. 1a). The methylation of four CpG sites in LINE-1 elements was chosen as a surrogate marker for global genomic DNA methylation [28]. Figure 1d shows that LINE-1 methylation decreased significantly upon treatment with 5 azadC concentrations as low as $1 \mathrm{nM}(P$ value $<0.05)$. This demethylating effect was dose-dependent up to the EC50 measured at day $7(100 \mathrm{nM})$.

Altogether, our data revealed that $5 \mathrm{azadC}$ displays an anti-invasive effect in a 3D metastatic melanoma model at low concentrations. This effect is correlated to its DNA demethylating action but not to its anti-metabolic and cytotoxic properties, as deduced from lack of effect of araC.

\section{DNA methylation modulation by $5 \mathrm{azadC}$ reactivates miR- 199a-3p}

To shed light on the mechanisms involved in the 5azadC-associated anti-invasive effect, we searched for genes and in particular miRs that were hypermethylated in the metastatic cell line WM-266-4 and which expression could be restored by promoter demethylation. Using data from a genome-wide DNA methylation analysis (BeadChip Illumina $450 \mathrm{~K}$, data not shown), we compared the methylation profile of WM-266-4 (metastatic cells) and its non-invasive counterpart WM-115 cell lines (derived from the primary tumour of the same patient). All genes and miR hypermethylated in WM-266-4 cell line with a difference of at least $20 \%$ on three CpGs were selected. Among the 68 hypermethylated miRs, two were chosen for further analysis since they were previously described as playing a role in cell invasion/migration processes: MIR-155HG [29] and MIR-199A2 [30, 31]. 
A

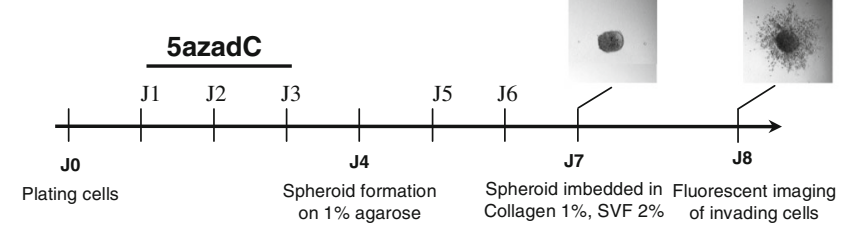

B $\quad 5$ azadC nM
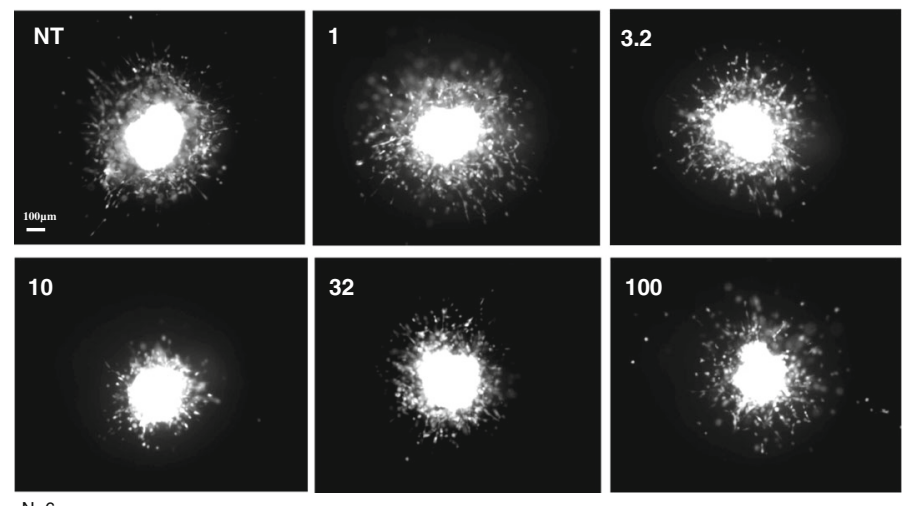

C

Mean Invasion Index

D

LINE-1 methylation level
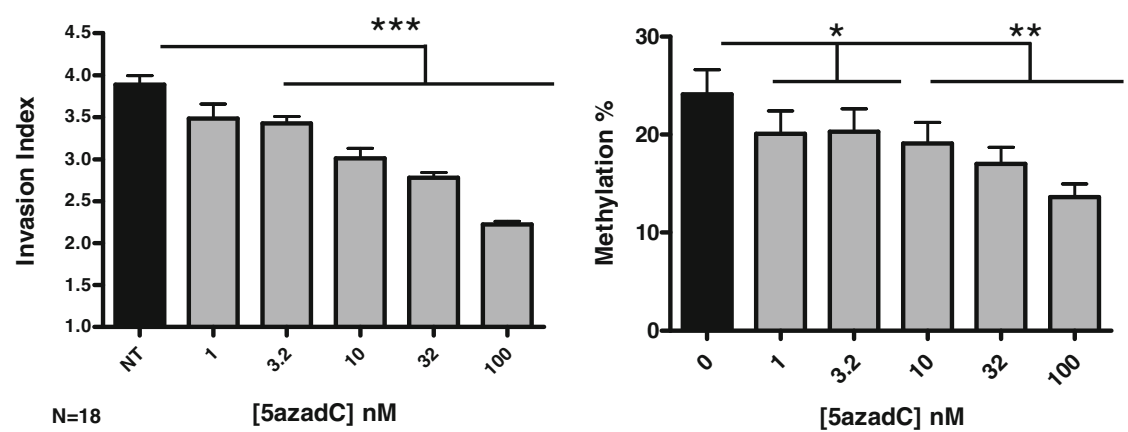

E
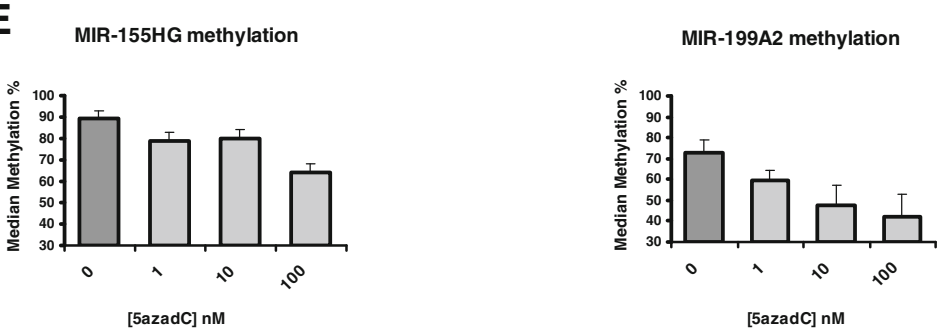

F
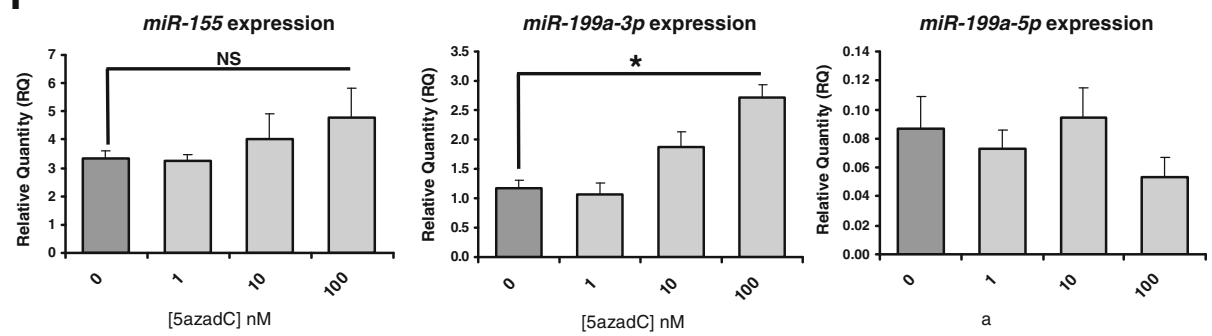

Fig. 1 (See legend on next page.) 
(See figure on previous page.)

Fig. 1 Effect of 5 azadC on 3D invasion properties, DNA methylation and specific miR expression in WM-266-4 GFP cells. a Experimental scheme for spheroid invasion assays. $\mathbf{b}$ Six spheroids were analysed for each 5azadC concentration as indicated. Photos shown in a are representative of one spheroid per condition. c Histogram representing the mean invasion index (invasion area/spheroid initial area) calculated for 18 spheroids. d Mean methylation percentage for five CpG sites in LINE-1 sequences. e Specific MIR-155HG and MIR-199A2 promoter demethylation was evaluated on spheroids after 5 azadC treatment. Median methylation of all the CpG sites is represented. $\mathbf{f}$ RT-qPCR analysis of mature miR-155, miR-199a-3p and miR199a-5p. The relative quantity $(\mathrm{RQ})$ was calculated using $R N U 6 B$ gene as control. All experiments were performed in triplicate. SEM are shown and ${ }^{*} P$ value $<0.05,{ }^{* *} P$ value $<0.01,{ }^{* * *} P$ value $<0.001$. NS not significant

The methylation status of CpG sites in the promoter of these two miRs was examined after 5 azadC treatment by DNA bisulfite conversion followed by pyrosequencing. For MIR-155HG, CpG sites were found hypermethylated in the non-treated WM-266-4 GFP spheroids with a median methylation of $88 \%$ (Fig. 1e). Upon 5azadC treatment, the decrease of methylation was comprised between 10 and 23\%. For the MIR-199A2 promoter, CpG sites were found methylated at $73 \%$ in non-treated WM-266-4 GFP spheroids and started to be demethylated from $1 \mathrm{nM}$ of 5azadC. A maximum demethylation of $31 \%$ was reached at $100 \mathrm{nM}$ (Fig. 1e).

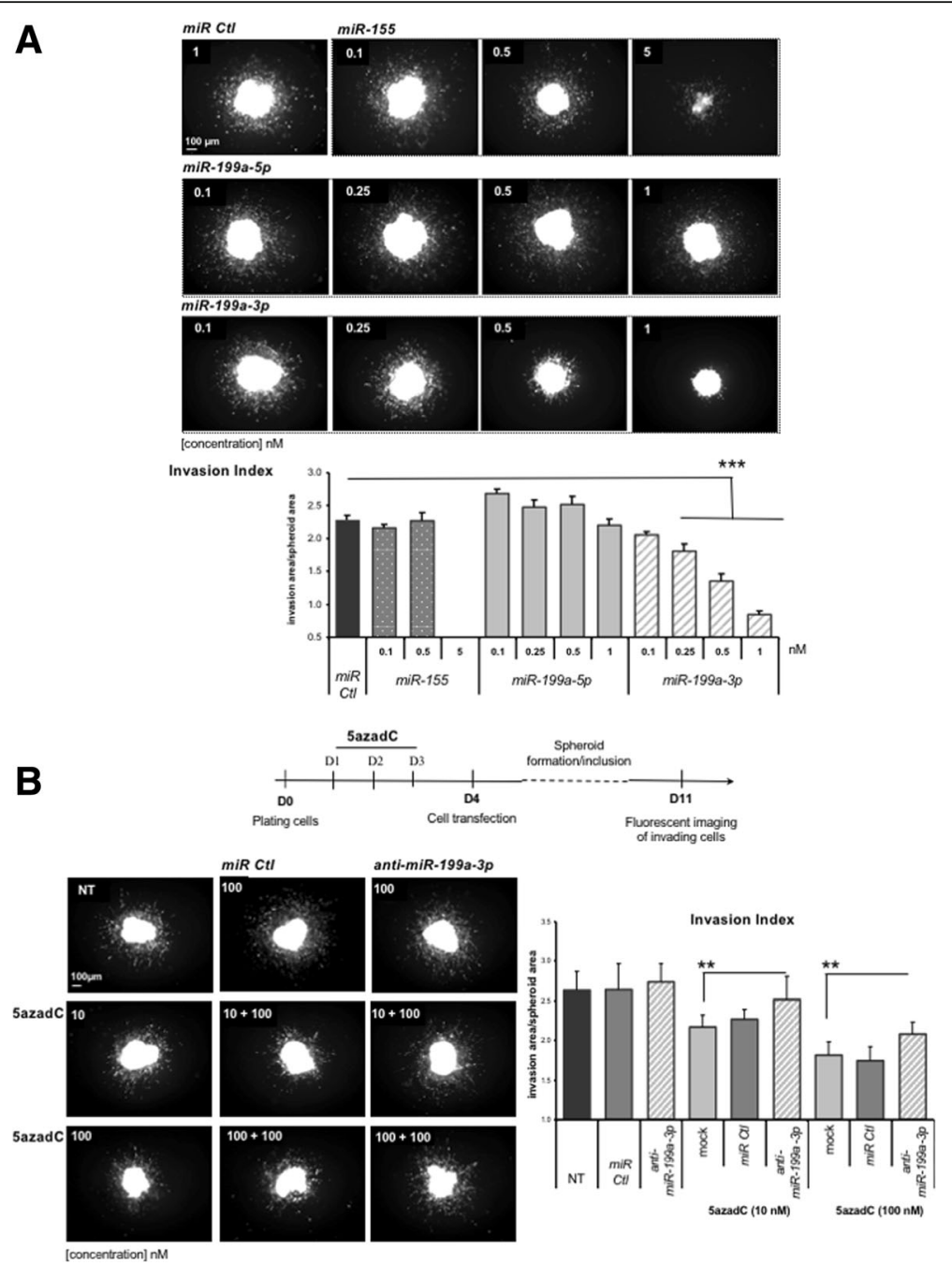

Fig. 2 Effect of miR overexpression on 3D metastatic melanoma cell invasion. a MiR-155, miR-199a-3p and miR-199a-5p were individually transfected, at the indicated concentration, in WM-266-4 GFP cells $48 \mathrm{~h}$ before spheroid formation. Images shown are representative of one spheroid per condition. The mean invasion index and SEM of two independent experiments are shown. b Top panel: main experimental steps for 5 azadC treatments and anti-miR199a-3p transfection before performing the 3D-invasion assay. Representative images of spheroids for each condition and invasion index quantification are presented. The mean index for 12 spheroids and SEM are shown. ${ }^{*} P$ value $<0.05,{ }^{*} P$ value $<0.01,{ }^{* *} P$ value $<0.001$, ND not determined, $\mathrm{Ctl}$ control and NT not treated 
To correlate this DNA demethylation with the respective RNA expression, mature miR levels were monitored in parallel. A dose-response increase of miR-155 expression was found upon $5 \mathrm{azadC}$ treatment without statistical significance (Fig. 1f). Regarding miR-199a, two mature miR species are known to be derived from the miR-199a precursor: $m i R-199 a-5 p$ and $m i R-199 a-3 p$, the former being 13 times less expressed than the latter (Fig. 1f, RQ to RNU6B of $0.086 \pm 0.023$ vs. $1.16 \pm 0.14$ ). A significant RNA re-expression of nearly threefold was found with $100 \mathrm{nM}$ of $5 \mathrm{azadC}$ for $m i R-199 a-3 p$, while no significant difference was observed for miR-199a-5p (Fig. 1f).

Hence, these results showed that low concentrations of 5azadC lead to DNA demethylation of specific hypermethylated miR promoters in metastatic melanoma WM-266-4 GFP cells. More specifically, MIR-199A2 promoter demethylation was correlated with the re-expression of its mature form miR-199a-3p.

\section{MiR-199a-3p impairs melanoma cell invasiveness and is involved in the anti-invasive effect of 5azadC}

Next, the effect of miR-199a-3p re-expression was evaluated in the metastatic melanoma 3D invasion assay. A miR-199a-3p mimetic was transiently expressed in WM-266-4 GFP cells $48 \mathrm{~h}$ before spheroid formation (Fig. 2). A non-relevant miR ( $m i R C t l$ ), $m i R-155$ and miR-199a-5p were used as controls (Fig. 2a and Additional file 1: Figure S4A). MiR-155 induced a cytotoxic effect starting from low concentrations $(5 \mathrm{nM})$, as already described by Levati et al. [32]. A drastic reduction of cell invasion was found at very low concentrations of miR-199a-3p (Fig. 2a). This anti-invasive effect started from $0.25 \mathrm{nM}$ and reached a maximum at $1 \mathrm{nM}$ $(P$ value $<0.001)$. It was specific to this miR, since its complementary counterpart, miR-199a-5p, did not induce such a decrease of the invasion index (Fig. 2a). These observations demonstrated that miR-199a-3p alone was able to suppress the 3D invasion ability of WM-266-4 GFP cells, suggesting that its re-expression induced by DNA demethylation can contribute to the anti-invasive effect of 5azadC.

To confirm this hypothesis, miR-199a-3p expression was knocked down in 5azadC-treated cells using a specific anti-miR-199a-3p (Fig. 2b and Additional file 1: Figure $\mathrm{S} 4 \mathrm{~B})$. The $3 \mathrm{D}$ anti-invasive ability of $5 \mathrm{azadC}$ was significantly reduced, and the invasion index was partially restored (Fig. 2b). These findings support our hypothesis that miR-199a-3p plays a role in the 5 azadC-mediated anti-invasive effect.

\section{MET targeting by miR-199a-3p is not sufficient to affect 3D cell invasion}

To better understand the role of miR-199a-3p in the invasion process, the combination of bioinformatics and

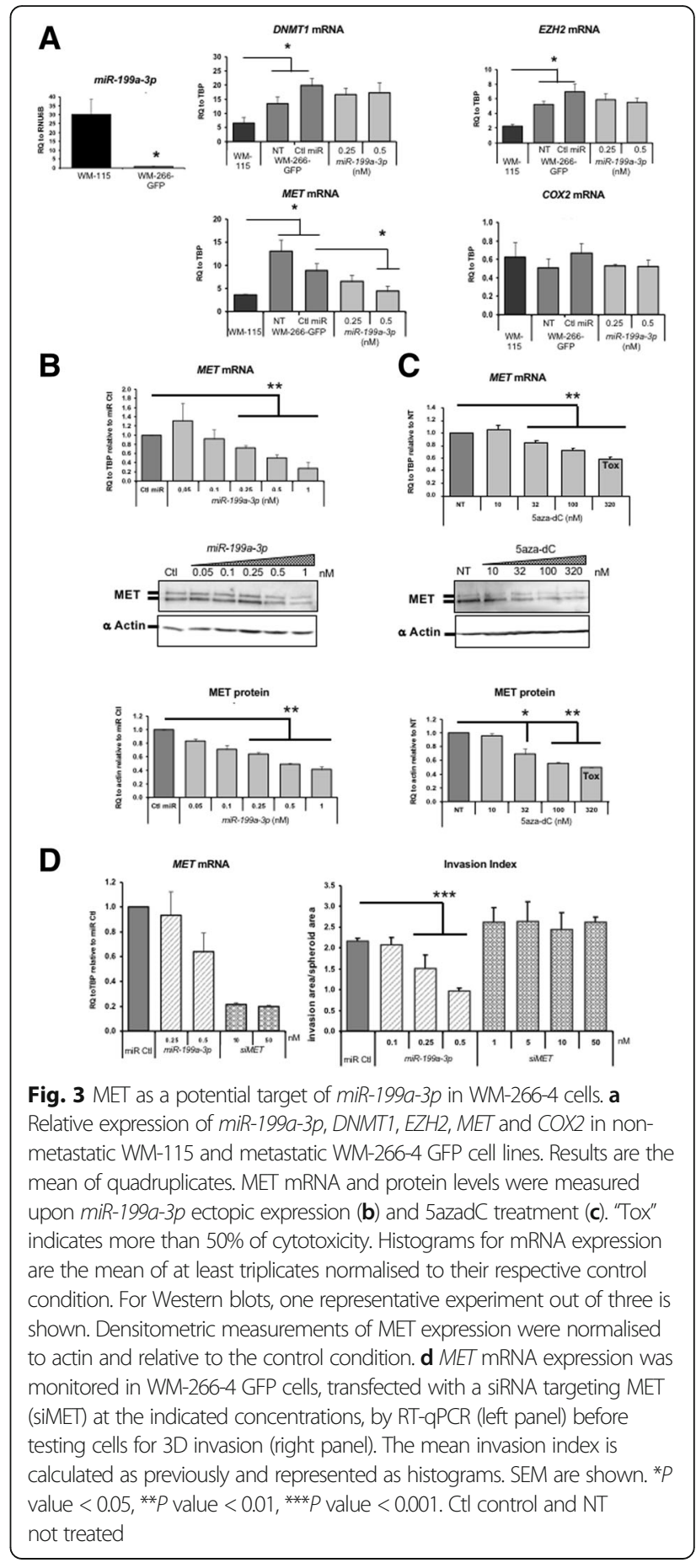

bibliographic approaches was used to identify its target genes. Among the 41 validated mRNAs targets, retrieved in the miRWalk database (http://zmf.umm.uni-heidelberg.de/apps/zmf/mirwalk2/), ten were related to the Gene Ontology (GO) terms of cell motion, cell migration and motility according to DAVID bioinformatics tool [33] (Table S2). In particular, two were already described to be highly expressed in melanomas and 

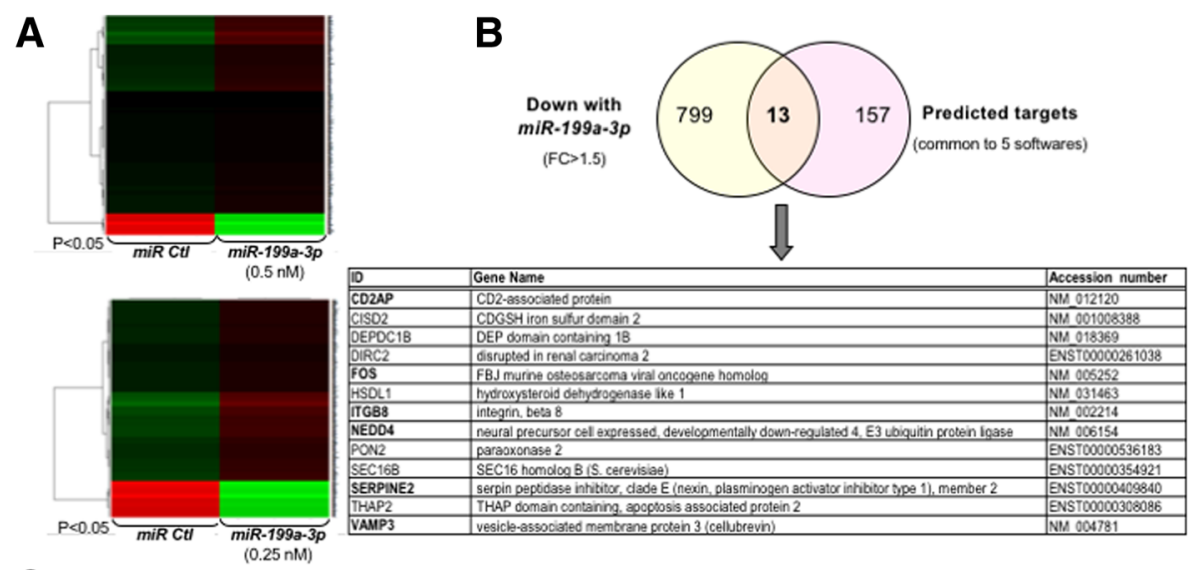

C
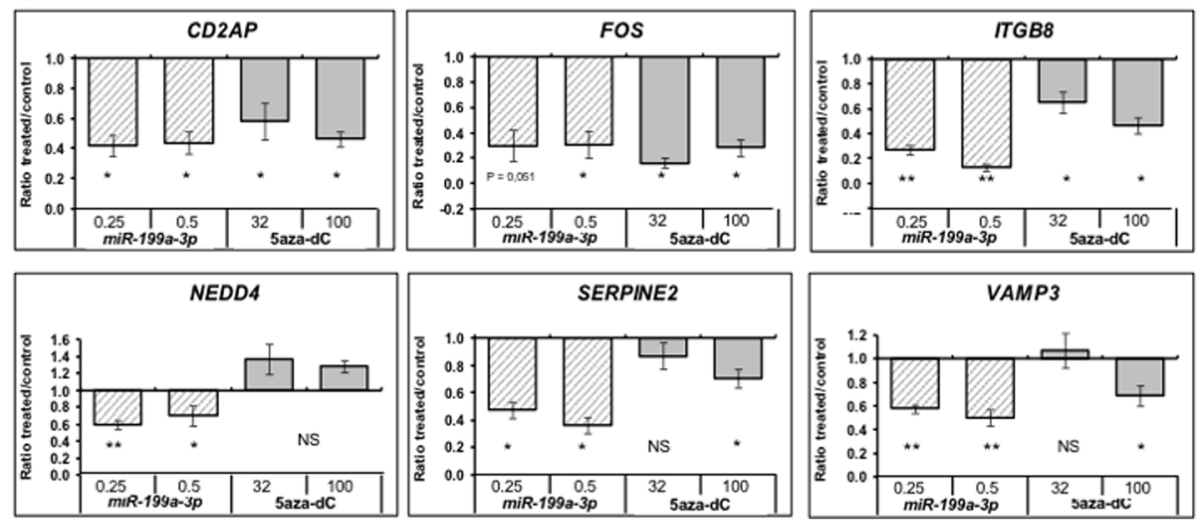

Fig. 4 Deregulated pathways and candidate target genes of miR-199a-3p. a Transcriptomic analysis of WM-266-4 GFP cells transfected with miR199a-3p identified differentially expressed genes with a FC > 1.5. Heatmaps represented all deregulated genes for the two conditions $(0.25$ and $0.5 \mathrm{nM}$ of miR-199a-3p). P value cutoff is FDR corrected. $\mathbf{b}$ Venn-diagram of all downregulated genes with a FC > 1.5 for miR-199a-3p-transfected cells (812 genes), crossed with its predicted targets (common to five different softwares). The table summarises the 13 retrieved genes. Genes in bold were further analysed. c CD2AP, FOS, ITGB8, NEDD4, SERPINE2 and VAMP3 expression in WM-266-4 cells transfected with miR-199a-3p or treated with $5 \mathrm{azadC}$. The mean fold inhibition was calculated as the ratio of RQ in treated cells/RQ in the control for each gene. SEM are shown for triplicates. ${ }^{*} P$ value $<0.05,{ }^{* *} P$ value $<0.01$. NS not significant

involved in the regulation of cell invasion: PTGS2, also known as COX2 [34], and MET oncogene [30]. In addition, we chose to study two major epigenetic regulators, DNMT1 (DNA methyltransferase 1) and EZH2 (enhancer of zeste homologue 2), since high levels of expression have been associated to melanoma aggressiveness [35]. As shown in Fig. 3a, expression levels of miR-199a-3p was significantly lower in the metastatic WM-266-4 cell line compared to the primary WM-115, while expression of DNMT1, EZH2 and MET were significantly higher and no significant differences were observed in the expression of COX2 mRNA (Fig. 3a). The higher expression of DNMT1 and EZH2 is concordant with previous observations in BRAF-mutated cells, such as it is the case for WM266-4, described to participate in the hypermethylated phenotype of metastatic melanoma cells ([36] and personal observations). In our model, miR-199a-3p ectopic expression, at concentrations inducing an anti-invasive effect (Fig. 2a), did not affect DNMT1,EZH2 and COX2 expression (Fig. 3a), but led to the decrease of $M E T$ mRNA and protein (Fig. 3a, b).

These results suggest an inverse relationship between miR-199a-3p and MET and thus a possible direct targeting of this oncogene by the miR. A similar decrease of $M E T$ was also observed upon 5azadC treatment (Fig. 3c), prompting us to hypothesise that re-expression of miR-199a-3p following DNA demethylation participates in the downregulation of MET. Nevertheless, MET knock down was not sufficient to inhibit 3D invasiveness of metastatic melanoma as shown in Fig. 3d. Therefore, MET depletion seemed not to be the major event leading to miR-199a-3p or 5azadC anti-invasive effect in our model.

\section{MiR-199a-3p and 5azadC deregulate common genes involved in invasion/migration pathways}

To identify other genes targeted by miR-199a-3p and involved in invasion processes, we compared global gene 


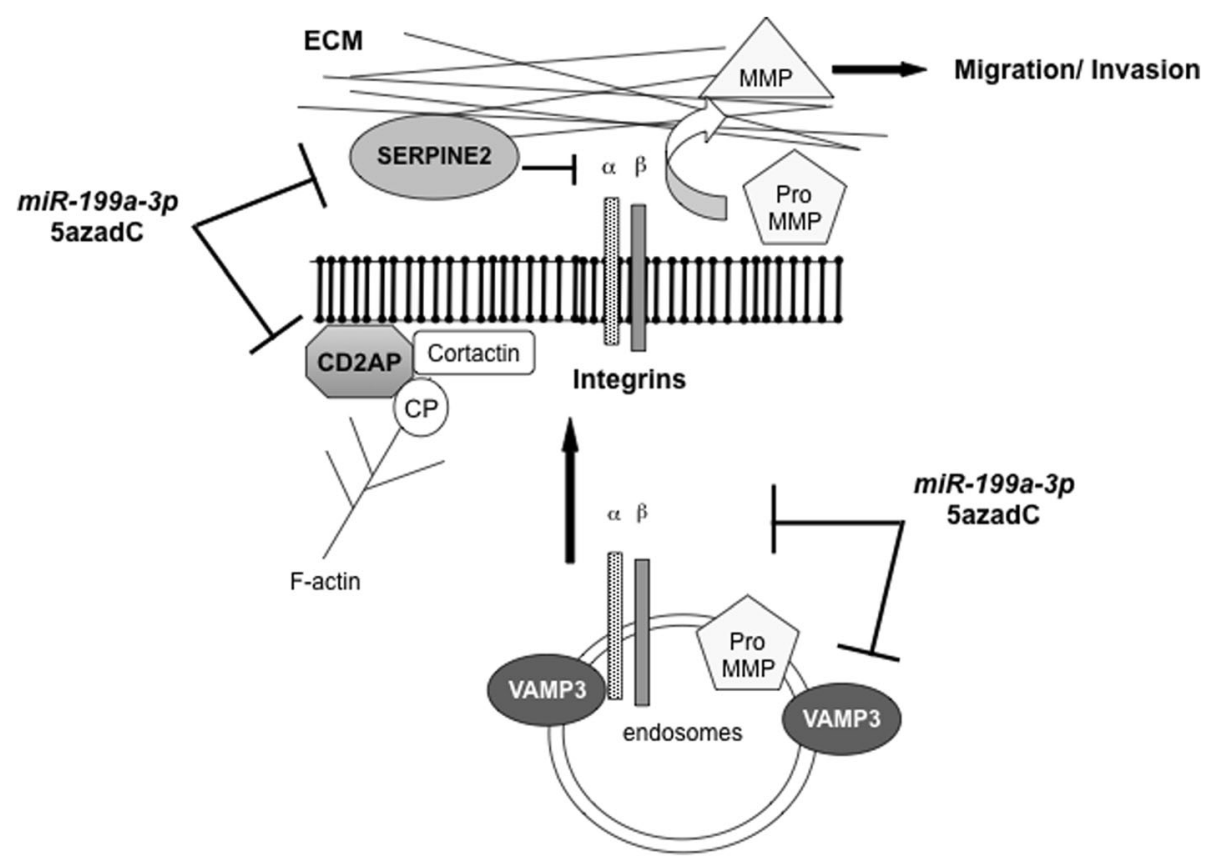

Fig. 5 Schematic representation of common downregulated genes by miR-199a-3p and 5azadC. Genes predicted as targets of miR-199a-3p and commonly downregulated by miR-199a-3p and 5azadC are highlighted in bold. ECM extracellular matrix, MMP matrix metalloproteinase, VAMP3 vesicle-associated membrane protein 3, CD2AP CD2-associated protein, CP capping protein, SERPINE2 serine protease inhibitor nexin 2

expression in miR-199a-3p vs control miR transiently transfected cells by transcriptomic microarrays. The differential gene expression analysis, using a fold change threshold $>1.5$ and a $P$ value $<0.05$ (Fig. 4a), revealed 812 downregulated genes.

Genes that were downregulated upon miR-199a-3p transfection were crossed with gene list of its predicted targets genes by five different softwares (DIANAmT, miRanda, miRDB, miRWalk and TargetScan) on the website: http:// zmf.umm.uni-heidelberg.de/apps/zmf/mirwalk2/ (as detailed in the Additional file 1). Among 170, thirteen genes were retrieved (Fig. 4b). Six of them, CD2AP, FOS, ITGB8, NEDD4, SERPINE2 and VAMP3 (highlighted in bold), belong to cell-signalling or cell migration/adhesion pathways and were chosen for further validation. The ectopic expression of miR-199a-3p reduced their expression at the mRNA levels with fold changes ranging from 1.5 to 8 (Fig. 4c). Noteworthy, five out of these genes were also downregulated upon 5azadC treatment (Fig. 4c). CD2AP is a membrane scaffold protein that has a role in dynamic actin assembly and lamellipodia formation. ITGB8, a $\beta$-integrin, connects adhesive proteins in the ECM to the intracellular actin cytoskeleton mediating cell adhesion and migration. SERPINE2, a member of the serine protease inhibitor nexin superfamily, contributes to the invasion in solid tumours and more specifically in colorectal cancers bearing KRAS or BRAF mutations [37]. VAMP3, an endosomal SNARE protein, is involved in vesicular trafficking and exocytosis of matrix metalloproteinases MMP2 and MMP9 and $\beta$-integrins that are required for cell motility. Finally, FOS forms heterodimers with JUN to reconstitute the transcription regulator AP-1, which participates in skin tumour development by controlling a wide range of cellular processes, including cell migration. Altogether, these results highlighted that $5 \mathrm{azadC}$ and miR-199a-3p impact on metastatic melanoma invasion properties by downregulating common effectors of this multistep biological process. Figure 5 schematically summarises the possible interplay between the four proteins, CD2AP, ITGB8, SERPINE2 and VAMP3, in the cell migration process, based on the data in the literature.

\section{Both 5azadC and miR-199a-3p alone impair lung metastasis formation by WM-266-4 cells in vivo}

To test the anti-tumour effect of $5 \mathrm{azadC}$ and $m i R-199 a-3 p$, in vivo experiments were performed using a melanoma model for metastasis formation in the lungs (Fig. 6a). SCID mice were injected in the tail vein with WM-266-4 cells previously treated with $100 \mathrm{nM} 5 \mathrm{azadC}$ or transfected with 0.5 or $1 \mathrm{nM}$ of miR-199a-3p, compared to non-treated or miR-Ctl-transfected cells, respectively. Lungs were collected 21 days after the injection, and metastasis numbers and area were counted after tyrosinase staining (Fig. 6a). A significant reduction of both parameters was observed upon 5azadC treatment (Fig. 6b). A similar anti-metastatic effect was observed with miR-199a-3p, despite a higher observed heterogeneity (Fig. 6c). These in vivo experiments validate $m i R-199 a-3 p$ as an anti-tumour effector and the potential interest of re-expressing this miR or using the 

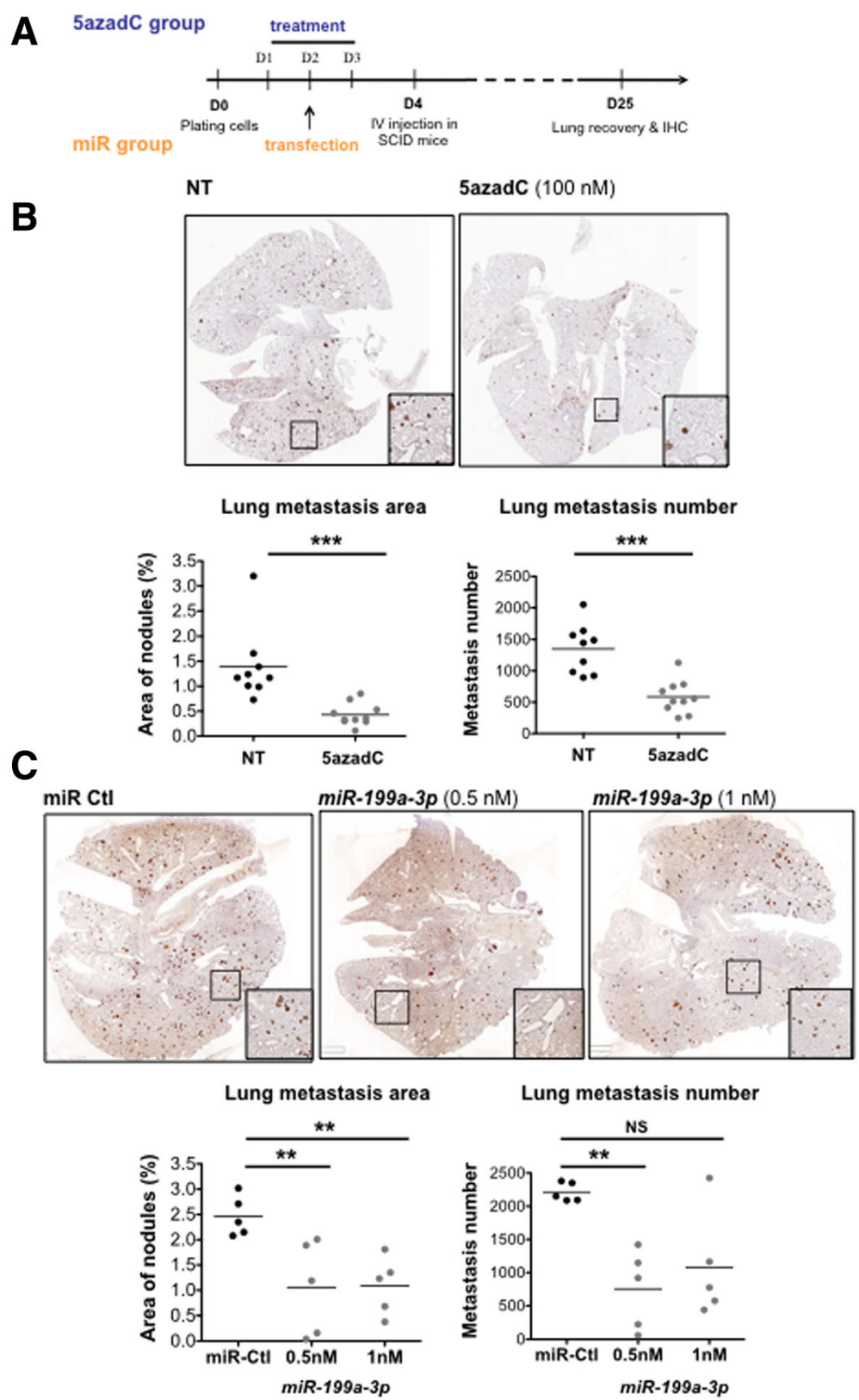

Fig. 6 In vivo effect of 5azadC and miR-199-3p expression on WM-266-4 lung metastasis. a Experimental outline: WM-266-4 cells treated or not with $100 \mathrm{nM}$ of $5 \mathrm{azadC}$ for 3 days or transfected with miR-199-3p mimetic at 0.5 or $1 \mathrm{nM}$ for $48 \mathrm{~h}$ were injected in the tail vein (IV) of SCID mice. Lungs were recovered for immunohistochemical analysis 21 days after injection. $\mathbf{b}$ A representative image of the stained lung is shown for each group. Plots representing the percentage area and the total number of metastasis on one slice for each mouse in non-treated (NT) and 5azadC-treated are shown for two independent experiments. Medians are shown. c Same as in B for miR Ctl and miR-199-3p tested at two concentrations, 0.5 and $1 \mathrm{nM}$. ${ }^{* *} P$ value $<0.01,{ }^{* * *} P$ value $<0.001$

demethylating drug $5 \mathrm{azadC}$ to limit metastasis formation in melanoma cells presenting a hypermethylated MIR-199A2.

\section{Promoter methylation status and expression of MIR- $199 \mathrm{~A} 2$ in patient samples}

To further validate the importance of MIR-199A2 DNA methylation in human melanoma progression, we analysed The Cancer Genome Atlas (TCGA) RNA-seq and
DNA methylation datasets available for skin cutaneous melanoma (SKCM) tumours on the UCSC cancer genomics browser website. In Fig. 7a, DNA methylation levels of the MIR-199A2 promoter were compared according to the tumour site location. This locus was found significantly ( $P$ value $<0.01)$ less methylated in the regional cutaneous tissues (RCT) than the primary tumours (PT) of the cohort, while an increase in its 


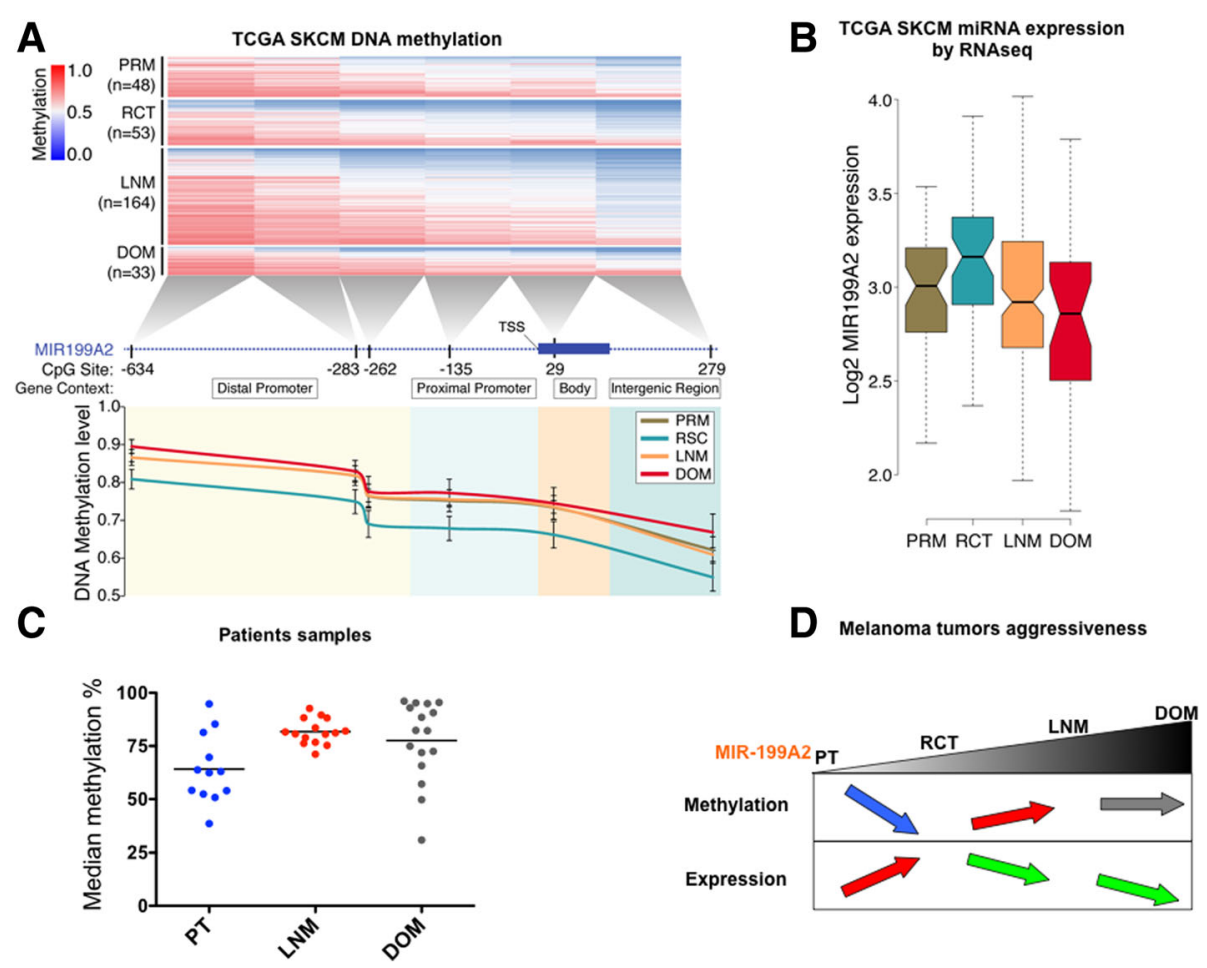

Fig. 7 Patient sample analysis and schematic representation of the role of miR-199-3p in melanoma aggressiveness. a DNA methylation level of six consecutive CpG sites in the genomic vicinity of MIR199A2 gene. TCGA SCKM data was classified according to the melanoma tissue type. b microRNA199A2 expression according to melanoma tissue types. c DNA methylation of Mir-199A2 were analysed in formaldehyde-fixated samples from 12 PT, $15 \mathrm{LN}$ and $16 \mathrm{DM}$ (10 bowel and 6 liver) samples. Median methylation of all the CpG sites is represented. $\mathbf{d}$ Schematic representation of MIR199A promoter methylation and expression as a function of the clinical stages. Blue arrows indicate a decrease in the DNA methylation and red arrows an increase. Green arrows indicate a decrease in the miR expression and red arrows an increase

methylation was observed in the regional lymph nodes metastases (LNM) in comparison to the RCT ( $P$ value < 0.05). Consistently, the expression of $m i R-199 a$ was found increased in the RCT, where it was less methylated, and decreased in the LNM and distant organ metastases (DOM), where it was more methylated ( $P$ value $<0.01$; Fig. 7b). We then confirmed these data on a cohort of 43 melanoma patients (Fig. 7c). These observations suggested that this locus is submitted to a tight epigenetic modulation during melanoma progression (Fig. 7d): demethylation at local stages (RCT), methylation and decreased expression at stages that start to disseminate (LN) and maintained at DMs. In summary, these data confirm that MIR-199A2 is regulated by DNA methylation of its promoter in patients and this methylation varies according to the disease stages. In addition, we observed that the MIR-199A2 promoter is commonly methylated in cells derived from patients bearing the most aggressive forms of the tumour compared to the primary counterparts with few exceptions (Additional file 1: Figure S5).

Taken together, these data support our hypothesis that MIR-199A2 hypermethylation can be important at an early stage for the acquisition of an invasive phenotype by the melanoma tumour cells.

\section{Discussion}

Epigenetic therapies are very promising strategies to fight cancer, since epigenetic modifications are reversible and can be chemically modulated. In particular, promoters' demethylation by 5-azaC or 5-azadC induces the re-expression of several genes or miRs in cancer cells inducing an anti-cancer effect [27]. To date, few clinical trials using epigenetic drugs have been conducted in metastatic melanoma; a recent example is the combination of 5azadC and temozolomide [38, 39]. Yet, epigenetic modifications and, in particular, genomic hypermethylation of $\mathrm{CpG}$ islands and miR modulation were shown to sign metastatic melanoma progression [40, 41].

To the best of our knowledge, few reports explored the impact of $5 \mathrm{azadC}$ on the cellular mechanism involved in metastatic melanoma, particularly on cell invasiveness, and its potential use as an anti-metastatic drug [23]. To address this question and better mimic the tumour behaviour in vivo, we developed a 3D cell invasion assay using spheroids embedded in a matrix of collagen I, the principal component of the extracellular matrix (ECM). Growing evidence showed that cancer 
cell spheroids reproduce various traits of micro-tumours, thus providing a better model for assessing tumour invasion [26]. Using the metastatic melanoma WM-266-4 GFP cell line, we showed for the first time that low-cytotoxic concentrations of 5azadC inhibit 3D cell invasion in vitro. In addition, this effect was clearly associated with a global and promoter-specific DNA demethylation. These observations are in complete agreement with Rajaii et al.'s results on uveal melanoma cells, describing that low concentrations of 5azadC reduced both 2D invasion and clonogenicity [23]. Our results in metastatic melanoma cells confirm also the anti-cancer potential of using low doses of the DNA demethylating agents in solid tumours as for haematological cancers $[27,38,42]$.

To further elucidate the mechanisms underlying the phenotypical changes induced by $5 \mathrm{azadC}$, we specifically investigated the modifications on miR methylation and expression. MiRs are post-transcriptional regulators that have a broad spectrum of cellular regulation by targeting multiple genes and pathways, including those required for metastatic melanoma progression like cell invasion and migration (reviews [40]). Moreover, their expression or DNA methylation signatures are studied as reliable markers for diagnosis and prognosis of cancer [10]. Here, two miRs, MIR-155HG and MIR-199A2, implicated in migration and invasion processes, were found hypermethylated in the metastatic melanoma cell line (WM-266-4) compared to its primary counterpart (WM-115). MiR-155 is an important multi-functional miR involved in the development of multiple solid tumours and is transcriptionally regulated by DNA methylation [29]. However, 5azadC-induced demethylation of MIR-155HG promoter ( $>20 \%$ ) was not sufficient to significantly reactivate $m i R-155$ expression in our metastatic melanoma cell model. Moreover, its ectopic re-expression in the WM-266-4 cells induced a cytotoxic effect rather than an anti-invasive effect. Thus, we focused on MIR-199A2 that was found hypermethylated in the WM-266-4 cell line and both demethylated and reactivated by 5 azadC.

Few reports identified epigenetically modulated miRs in melanomas (reviews [40, 41]). For the first time, we report that MIR-199A2 locus is under epigenetic regulation in a metastatic melanoma model and it is reactivated by promoter demethylation by $5 \mathrm{azadC}$. In addition, nanomolar concentrations of miR-199a-3p impairs 3D cell invasion, unlike miR-199a-5p. Furthermore, the anti-invasive effect of $5 \mathrm{azadC}$ was counterbalanced by a specific antago-miR suggesting that miR-199a-3p takes part of the anti-invasive response induced by $5 \mathrm{azadC}$. Importantly, the transient expression of miR-199a-3p in WM-266-4 cells reduced significantly their metastatic potency in vivo, supporting an anti-cancer effect of this miR.
These results are somewhat contradictory to those reported by Pencheva et al. (2012) [43], describing that the cooperation between three miRs, miR-199a-3p, $m i R-199 a-5 p$ and $m i R-1908$, promoted metastasis by impairing two proteins, APOE and DNAJA4, involved in tumour cell invasion and in angiogenesis. This discrepancy can be explained as follows. First, the cellular models used are significantly different. Pencheva et al. selected for their study the most in vivo metastatic subclones of MeWo and A375, two metastatic melanoma cell lines bearing BRAF wild-type and BRAFV600E mutation, respectively. These subclones overexpressed $m i R-199 a-3 p$ and miR-199a-5p in different proportions. The A375 subclones, bearing a mutated BRAF, as it is the case of the WM266-4 cell line, overexpressed much higher levels of miR-199a-5p $(\times 10,000)$ than of $m i R-199 a-3 p(\times 10)$, strongly suggesting that mainly the miR-199a-5p supported the pro-metastatic effect that was observed by the authors in these subclones. In agreement with this hypothesis, we observed a reversion of the anti-invasive effect of miR-199a-3p when combined with increasing amounts of the complementary miR-199a-5p mimetic (data not shown), revealing a sponge effect of the latter. Second, Pencheva et al. performed in vivo experiments with the combination of the three LNA anti-miRs, targeting miR-199a-3p, $m i R-199 a-5 p$ and $m i R-1908$ but not with individual ones or the miR-199a-3p mimetic alone. Thus, it cannot be excluded that the balance between each miR level can affect the tumour development. Moreover, cumulative evidences have shown that miR-199a has important but versatile functions in tumourigenesis depending on the nature and the stage of the cancer [31]. Indeed, Yang et al. found that low expression levels of miR-199a-5p in tumour melanoma tissue samples from patients were associated to the advances of tumour stage [44]. Their observations in B16 melanoma cells and in tumour xenografts seem to indicate that $m i R-199 a-5 p$ is a tumour suppressor, via direct targeting of HIF-1 $\alpha$. Third, our findings in metastatic melanoma are in agreement with an increasing number of observations in other cancer types. In testicular tumour, MIR-199A2 hypermethylation was linked to malignancy progression [45]. In this model, miR-199a re-expression led to the suppression of cell growth, cancer migration, invasion and metastasis in vitro and in vivo. Similar observations were made in non-small cell lung cancer, colorectal cancer and breast cancer cell lines [46]. A tumour-suppressive role of this miR was also demonstrated in papillary thyroid carcinoma, in which miR-199a-3p was shown to be likely regulated by the BRAFV600E oncogene [47]. Similar anti-cancer functions were described in renal cell carcinoma [48], endometrial carcinoma [49] and prostate cancer [50]. 
Finally, to better understand the mechanisms involved in the miR-199a-3p-mediated anti-invasive effect in metastatic melanoma, its target genes were investigated. The proto-oncogene MET was found overexpressed in the metastatic vs. primary cell line. Despite its downregulation by both $m i R-199 a-3 p$ and 5 azadC, its specific inhibition was not sufficient to impair 3D metastatic melanoma invasion (Fig. 4). Next, by a transcriptomic and bioinformatic approach, we observed that miR-199a-3p targeted several genes involved in key pathways for cell migration/invasion. This alteration of multiple targets can better explain the in vitro anti-invasive and in vivo anti-metastatic effects of the miR. Interestingly, 5azadC also downregulated common effectors of this multistep biological process (Fig. 5). In addition, analysis of TCGA SKCM datasets supports a tight modulation of $M I R-199 A 2$ promoter methylation and $m i R-199 a$ expression during melanoma progression, with local stages (RCT) presenting a promoter demethylation and an increased expression, whereas disseminated tumours (LN) show a MIR-199A2 hypermethylation concomitant to a decreased repression (Fig. 7). These observations were further confirmed on an experimental cohort of primary melanoma, lymph node and distant metastasis. Altogether, these observations can explain the different effects found in the literature for miR-199a-3p depending on its targets and strongly promote the interest of its reactivation at early melanoma stages that start to locally disseminate.

\section{Conclusions}

In conclusion, we proved that slightly cytotoxic doses of 5 azadC are translated into an anti-invasive effect in a BRAF-mutated metastatic melanoma model. This effect is in part associated with MIR-199A2 promoter demethylation and re-expression of its specific mature form $m i R-199 a-3 p$. Indeed, this miR on its own impairs 3D cell invasion by simultaneously targeting multiple genes involved in adhesion/migration processes. This effect was further confirmed in an in vivo xenograft model with transiently transfected cells, thus supporting its implication in metastatic melanoma aggressiveness. Finally, the clinical relevance of our results is confirmed by the analysis of the TCGA cancer skin cohort and 43 patient samples, showing that MIR-199A2 promoter methylation and expression is finely tuned during melanoma progression. Hypermethylation of its promoter was found associated with LN metastasis stages before distant dissemination (Fig. 7), suggesting its expression extinction could be involved in metastasis progression. Taken together, our findings support the use of epigenetic drugs or miR mimetic-based therapeutic strategies, either alone or in combination, for limiting metastatic dissemination of melanomas. Concordantly, a combination of
5 azadC with temozolomide was recently investigated in a phase I/II trial and proven to be safe and improve response and overall survival rates [38].

\section{Materials and methods}

The details of the protocols, including primer sequences, are reported in Additional file 1.

\section{Human cell lines}

The WM-115 and WM-266-4 cell lines were purchased from the European Collection of Authenticated Cell Culture (ECACC), were subjected to cell line authentication via short tandem repeat profiling and were grown up to a maximum of 20 passages and for fewer than 6 months following resuscitation in our laboratory. The WM-266-4 GFP cell line was obtained by transduction with WPXLd, a lentiviral vector encoding GFP, and selected by flow cytometry. Additionally, four primary melanoma cell lines (WC00060, WC00062, WC0008 and WC00081) were purchased from the Coriell Cell Repository (Coriell Institute for Medical Research, Camden, NJ, USA).

\section{Drug treatments}

5-Aza-2' -deoxycytidine (5azadC, decitabine) and cytarabine (araC) from Sigma-Aldrich (St Louis, USA) were dissolved in water at $10 \mathrm{mM}$ and frozen at $-20^{\circ} \mathrm{C}$ until use. Serial dilutions were made in culture media and added on cells seeded $24 \mathrm{~h}$ earlier at a density of $0.6 \times$ $10^{5}$ cells $/ \mathrm{mL}$ in a 12 -well plate. Treatment was repeated during 3 days, and cells were harvested at day 4 for further analysis.

\section{Cell viability assay}

The half effective concentration (EC50) of 5azadC or araC was determined at day 7 after the first treatment on WM-266-4 GFP cells using ATP-lite ${ }^{\mathrm{TM}}$ assay (Perkin Elmer, France) according to the manufacturer for cells cultured in 2D conditions. This protocol was also adapted for spheroids (3D conditions).

\section{D cell invasion assay}

WM-266-4 GFP (3000 cells/well) were allowed to form spheroids for 2 days on agarose $1 \%$ (Sigma-Aldrich, \#A95-39) coated in 96-well plates, leading to 300- $\mu \mathrm{m}$-diameter spheroids. Then, six different spheroids for each condition were individually embedded in EMEM media (Lonza, \#BE12-684F) containing 1\% of Bovine Collagen I (BD, \#354231) and 2\% SVF. The initial spheroid size (day 7) and the 24-h invasion area (day 8) were measured by fluorescent microscopy, using an Axiovert $200 \mathrm{M}$ device (5X Plan-Neofluor objective, Carl Zeiss, Germany). Fluorescent invasion areas were quantified using Image $(\mathrm{NIH})$ software on the sum of six Z-stacks images (20- $\mu \mathrm{m}$ interval) for each spheroid. 
Invasion index was obtained by normalising the invasion area at $24 \mathrm{~h}$ by the initial spheroid area: invasion area/ spheroid area (Additional file 1: Figure S1). Six individual spheroids were quantified for each condition, and three independent experiments were performed.

\section{Genomic DNA purification}

Paraffin-embedded tissue sections were microdissected, and DNA was purified using the ZR FFPE DNA MiniPrep (Zymo Research, Irvine, CA) as reported [51]. Genomic DNA from melanoma cell lines was extracted using DNAzol reagent (Life Technologies, Carlsbad, CA) as in [7].

\section{Bisulfite modification and pyrosequencing}

Quantitative DNA methylation analysis was performed by pyrosequencing of bisulfite-treated DNA as described in [52]. Two PCR were carried out for the five CpGs of interest on the miR-199a-3p locus.

PCR1: (1 CpG):

Forward: TTAGGGGTTGTATTTAGTTTTTTTT

Reverse: Biotine-ATTCATTACCAATTCCCCAATCTA

Pyrosequencing primer: GGTTGTGATTTTTAGTTTT GAYGTGGT

PCR2: (4 CpGs):

Forward: Biotine-GTTATTTTGGGGAGGTTTGGGTAT G

Reverse: CCCACTTCCTACCCAATTAAAAAAAA

Pyrosequencing primer:

CCATTTTATACACAAACCCATATCTAAAA ACAAACRATTCTAACRATCTCTCCAACRACAC AACRCATA

\section{Microarray expression and GO terms analysis}

RNA samples were extracted from cells transfected with miR-199a-3p miR mimetic as described in Additional file 1. Experiments were performed in three biological replicates for each condition and analysed on Affymetrix Human Gene 2.0 ST arrays. RMA normalisation was applied, and genes differentially expressed between treated and control conditions were determined choosing a threshold of $\log _{2}$ fold change at 0.5 and a $P$ value $<0.05$. $P$ value cutoff is FDR corrected [53]. Significantly enriched pathways were chosen using a threshold enrichment score $>2$. Data have been submitted to the Gene Expression Omnibus database.

\section{In vivo metastasis experiments}

The animals were handled and cared for in accordance with the Guide for the Care and Use of Laboratory Animals (National Research Council, 1996) and European Directive EEC/86/609, under the supervision of the authorised investigators. Un-anesthetised 7-week-old female SCID mice (Charles River Laboratories, Saint-
Germain-sur-l'Arbresle, France) were injected into the tail vein with $3 \times 10^{6}$ viable WM-266-4 cells in $200-\mu \mathrm{L}$ PBS, previously treated or not with $5 \mathrm{azadC}$ at $100 \mathrm{nM}$ $(3 \times 3$ days $)$ or transfected with $\mathrm{miR}$ negative control \#1 (miR Ctl) or miR-199a-3p mimic at 0.5 or $1 \mathrm{nM}$ during $48 \mathrm{~h}$. Each group constituted of 4-5 mice. For 5azadC treatment, two independent experiments were performed. Twenty-one days after injection, mice were dissected and lungs recovered, formalin fixed and paraffin embedded. Sections were stained with haematoxylin and eosin (H\&E). The number and area of macrometastasis were measured in total lung sections by immunostaining with Tyrosinase antibody Mob299-05 (1/500) (Diagnostic BioSystem, Pleasanton, CA-USA) on an Aperio Scanner (Leica) with Tissue Studio software (Definiens, Munich, Switzerland) for automatic segmentation and quantification of lesions. Statistics were performed using the Mann-Whitney test.

\section{The Cancer Genome Atlas skin cutaneous melanoma (TCGA-SKCM) dataset analysis}

The TCGA-SKCM DNA methylation, RNA-sequencing (RNAseq) and clinical datasets were accessed in June 2016. Wilcoxon-Mann-Whitney test was applied to determine the significance of DNA methylation or gene expression differences between primary and metastatic melanoma tissues.

\section{Melanoma patients included in the study}

Melanoma patients and healthy individuals were included in the study under protocols approved by the Western institutional review board. Informed consent was obtained from all subjects, and the experiments were performed according to the principles set out in the WMA Declaration of Helsinki and the NIH Belmont Report. Tissue specimens were coded according to HIPAA recommendations to ensure the confidentiality of the patients. The specimen cohort included paraffin-embedded tissues including nevi (NEV; $n=7$ ), primary melanomas (PRM; $n=16$ ), lymph node metastases (LNM; $n=15)$ and distant organ metastases (DOM; $n=20)$. Additionally, cell lines derived from LNM $(n=$ 11) were established at JWCI and included in the study. For each patient, median DNA methylation was calculated on five CpGs on the miR-199a-3p locus.

\section{Additional file}

Additional file 1: Table S1. Target sequences, PCR and sequencing primers used for pyrosequencing assays. Figure $\mathbf{S 1}$. Measurement of the invasion index. Figure S2. Cytotoxic effect of 5azadC on WM-266-4 and WM-266-4 GFP cells. Figure S3. Comparison of araC and 5azadC effects on cell viability and $3 \mathrm{D}$ invasion. Figure S4. MiRNA expression levels after transfection. Figure S5 (A) Mir-199A2 CpG methylation in cell lines. B) RTqPCR analysis of mature miR-199a-3p in cell lines. (DOCX $1759 \mathrm{~kb}$ ) 


\section{Abbreviations}

5aza: 5-Aza-cytidine; 5azadC: 5-Aza-deoxycytidine; araC: Cytarabine; CIMP: CpG island methylator phenotype; Ctl: Control; DNMT: DNA methyltransferase; DNMTi: DNA methyltransferase inhibitors; DOM: Distant organ metastases; EC50: Half effective concentration; LNM: Lymph nodes metastases; miR: MicroRNA; RCT: Regional cutaneous tissues; SKCM: Skin cutaneous melanoma; TCGA: The Cancer Genome Atlas

\section{Acknowledgements}

Not applicable.

\section{Funding}

This work was supported to P.B.A. by Centre National de la Recherche Scientifique (CNRS) [ATIP], Région Midi Pyrenées [Equipe d'Excellence and FEDER CNRS/Région Midi Pyrenées], Fondation InNaBioSanté, and PlanCancer2014 (NEPIG201401).

\section{Availability of data and materials}

In addition to the data available in the supplementary information files, datasets used and/or analysed during the current study are available from the corresponding author. Materials are also available on reasonable request.

\section{Authors' contributions}

CD conceived and performed the experiments and wrote the manuscript. AC characterised the DNA methylation, analysed the patient data and performed the experiments. $A D$ set up the $3 D$ invasion model. AD, FB and JT performed the pyrosequencing assays. AP and JR performed the in vivo experiments. DM contributed with the data analysis and DNA purification from melanoma tissues and cell lines. JR and GF participated in the design of the study. CE and PBA oversaw and designed the study and wrote the manuscript. All authors read and approved the final manuscript.

\section{Ethics approval and consent to participate}

Informed consent was obtained from all subjects, and experiments were performed according to the principles set out in the WMA Declaration of Helsinki and the NIH Belmont Report. Tissue specimens were coded according to HIPAA recommendations to ensure the confidentiality of the patients.

\section{Consent for publication}

Not applicable.

\section{Competing interests}

The authors declare that they have no competing interests.

\section{Publisher's Note}

Springer Nature remains neutral with regard to jurisdictional claims in published maps and institutional affiliations.

\section{Author details}

${ }^{1}$ FRE no. 3600 CNRS, Epigenetic Targeting of Cancer (ETaC), Toulouse, France. ${ }^{2}$ Cancer Research Center of Toulouse, CRCT, Toulouse, France. ${ }^{3}$ Department of Translational Molecular Medicine, John Wayne Cancer Institute, Providence Saint John's Health Center, Santa Monica, CA, USA. ${ }^{4}$ Laboratory for Functional Genomics, Fondation Jean Dausset - CEPH, Paris, France. ${ }^{5}$ Laboratory for Epigenetics and Environment, Centre National de la Recherche en Génomique Humaine, CEA, Evry, France. ${ }^{6}$ Institut de Recherche Pierre Fabre, CRDPF, Toulouse, France. 'UMR 1037 INSERM/Université Toulouse III, CRCT, Toulouse, France. ${ }^{8}$ Institut Pasteur CNRS UMR3523, Epigenetic Chemical Biology, Paris, France. ${ }^{9} \mathrm{CNRS}$-Pierre Fabre USR3388 ETaC, Toulouse, France.

\section{Received: 4 October 2018 Accepted: 17 December 2018}

\section{Published online: 16 January 2019}

\section{References}

1. Shain AH, Bastian BC. From melanocytes to melanomas. Nat Rev Cancer. 2016;16(6):345-58

2. Atkinson V. Recent advances in malignant melanoma. Intern Med J. 2017 ; 47(10):1114-21.
3. Azad N, Zahnow CA, Rudin CM, Baylin SB. The future of epigenetic therapy in solid tumours--lessons from the past. Nat Rev Clin Oncol. 2013;10(5):256-66

4. Conway K, Edmiston SN, Khondker ZS, Groben PA, Zhou X, Chu H, et al. DNA-methylation profiling distinguishes malignant melanomas from benign nevi. Pigment Cell Melanoma Res. 2011;24(2):352-60.

5. Sigalotti L, Covre A, Fratta E, Parisi G, Sonego P, Colizzi F, et al. Whole genome methylation profiles as independent markers of survival in stage iiic melanoma patients. J Transl Med. 2012;10(1):185.

6. Koga Y, Pelizzola M, Cheng E, Krauthammer M, Sznol M, Ariyan S, et al. Genome-wide screen of promoter methylation identifies novel markers in melanoma. Genome Res. 2009;19(8):1462-70.

7. Marzese DM, Scolyer RA, Huynh JL, Huang SK, Hirose H, Chong KK, et al. Epigenome-wide DNA methylation landscape of melanoma progression to brain metastasis reveals aberrations on homeobox D cluster associated with prognosis. Hum Mol Genet. 2014;23(1):226-38

8. Ecsedi S, Hernandez-Vargas H, Lima SC, Vizkeleti L, Toth R, Lazar V, et al, DNA methylation characteristics of primary melanomas with distinct biological behaviour. PLoS One. 2014;9(5):e96612.

9. Moran B, Silva R, Perry AS, Gallagher WM. Epigenetics of malignant melanoma. Semin Cancer Biol. 2018:51:80-8.

10. Besaratinia A, Tommasi S. Epigenetics of human melanoma: promises and challenges. J Mol Cell Biol. 2014.

11. Tellez CS, Shen L, Estecio MR, Jelinek J, Gershenwald JE, Issa JP. CpG island methylation profiling in human melanoma cell lines. Melanoma Res. 2009; 19(3):146-55.

12. Bonazzi VF, Irwin D, Hayward NK. Identification of candidate tumor suppressor genes inactivated by promoter methylation in melanoma. Genes, chromosomes \& cancer. 2009;48(1):10-21.

13. Jin SG, Xiong W, Wu X, Yang L, Pfeifer GP. The DNA methylation landscape of human melanoma. Genomics. 2015;106(6):322-30.

14. Thomas NE, Slater NA, Edmiston SN, Zhou X, Kuan PF, Groben PA, et al. DNA methylation profiles in primary cutaneous melanomas are associated with clinically significant pathologic features. Pigment Cell Melanoma Res. 2014;27(6):1097-105

15. Tanemura A, Terando AM, Sim MS, van Hoesel AQ, de Maat MF, Morton DL, et al. CpG island methylator phenotype predicts progression of malignant melanoma. Clin Cancer Res. 2009:15(5):1801-7.

16. Sarkar D, Leung EY, Baguley BC, Finlay GJ, Askarian-Amiri ME. Epigenetic regulation in human melanoma: past and future. Epigenetics. 2015;10(2): 103-21.

17. Micevic G, Theodosakis N, Bosenberg M. Aberrant DNA methylation in melanoma: biomarker and therapeutic opportunities. Clin Epigenetics. 2017; 9:34.

18. Sigalotti L, Covre A, Fratta E, Parisi G, Colizzi F, Rizzo A, et al. Epigenetics of human cutaneous melanoma: setting the stage for new therapeutic strategies. J Transl Med. 2010;8:56

19. Philippidou D, Schmitt M, Moser D, Margue C, Nazarov PV, Muller A, et al. Signatures of microRNAs and selected microRNA target genes in human melanoma. Cancer Res. 2010;70(10):4163-73.

20. Greenberg E, Nemlich Y, Markel G. MicroRNAs in cancer: lessons from melanoma. Curr Pharm Des. 2014;20(33):5246-59.

21. Gasque Schoof $C R$, Izzotti $A$, Jasiulionis MG, Vasques Ldos $R$. The roles of miR-26, miR-29, and miR-203 in the silencing of the epigenetic machinery during melanocyte transformation. Biomed Res Int. 2015;2015:634749.

22. Alcazar O, Achberger S, Aldrich W, Hu Z, Negrotto S, Saunthararajah Y, et al. Epigenetic regulation by decitabine of melanoma differentiation in vitro and in vivo. Int J Cancer J Int du cancer. 2012;131(1):18-29.

23. Rajaii F, Asnaghi L, Enke R, Merbs SL, Handa JT, Eberhart CG. The demethylating agent 5-AZA reduces the growth, invasiveness, and clonogenicity of uveal and cutaneous melanoma. Invest Ophthalmol Vis Sci. 2014:55(10):6178-86.

24. Noguchi S, Mori T, Igase M, Mizuno T. A novel apoptosis-inducing mechanism of 5-aza-2'-deoxycitidine in melanoma cells: demethylation of TNF-alpha and activation of FOXO1. Cancer Lett. 2015;369(2):344-53.

25. Lucarini V, Buccione C, Ziccheddu G, Peschiaroli F, Sestili P, Puglisi R, et al. Combining type I interferons and 5-aza-2'-deoxycitidine to improve antitumor response against melanoma. J Invest Dermatol. 2017;137(1):159-69.

26. Hirschhaeuser F, Menne H, Dittfeld C, West J, Mueller-Klieser W, KunzSchughart LA. Multicellular tumor spheroids: an underestimated tool is catching up again. J Biotechnol. 2010;148(1):3-15. 
27. Tsai HC, Li H, Van Neste L, Cai Y, Robert C, Rassool FV, et al. Transient low doses of DNA-demethylating agents exert durable antitumor effects on hematological and epithelial tumor cells. Cancer Cell. 2012;21(3):430-46.

28. Weisenberger DJ, Campan M, Long TI, Kim M, Woods C, Fiala E, et al. Analysis of repetitive element DNA methylation by MethyLight. Nucleic Acids Res. 2005:33(21):6823-36.

29. Levati L, Pagani E, Romani S, Castiglia D, Piccinni E, Covaciu C, et al. MicroRNA-155 targets the SKI gene in human melanoma cell lines. Pigment Cell Melanoma Res. 2011;24(3):538-50

30. Migliore C, Petrelli A, Ghiso E, Corso S, Capparuccia L, Eramo A, et al. MicroRNAs impair MET-mediated invasive growth. Cancer Res. 2008;68(24): 10128-36.

31. Gu S, Chan WY. Flexible and versatile as a chameleon-sophisticated functions of microRNA-199a. Int J Mol Sci. 2012;13(7):8449-66.

32. Levati L, Alvino E, Pagani E, Arcelli D, Caporaso P, Bondanza S, et al. Altered expression of selected microRNAs in melanoma: antiproliferative and proapoptotic activity of miRNA-155. Int J Oncol. 2009;35(2):393-400.

33. Huang d W, Sherman BT, Lempicki RA. Systematic and integrative analysis of large gene lists using DAVID bioinformatics resources. Nat Protoc. 2009:4(1):44-57.

34. Minisini AM, Pascoletti G, Intersimone D, Poletto E, Driol P, Spizzo R, et al. Expression of thymidine phosphorylase and cyclooxygenase-2 in melanoma. Melanoma Res. 2013;23(2):96-101.

35. Bachmann IM, Halvorsen OJ, Collett K, Stefansson IM, Straume O, Haukaas $\mathrm{SA}$, et al. EZH2 expression is associated with high proliferation rate and aggressive tumor subgroups in cutaneous melanoma and cancers of the endometrium, prostate, and breast. J Clin Oncol. 2006;24(2):268-73.

36. Hou P, Liu D, Dong J, Xing M. The BRAF(V600E) causes widespread alterations in gene methylation in the genome of melanoma cells. Cell Cycle. 2012;11(2):286-95.

37. Bergeron S, Lemieux E, Durand V, Cagnol S, Carrier JC, Lussier JG, et al. The serine protease inhibitor serpinE2 is a novel target of ERK signaling involved in human colorectal tumorigenesis. Mol Cancer. 2010;9:271.

38. Tawbi HA, Beumer JH, Tarhini AA, Moschos S, Buch SC, Egorin MJ, et al. Safety and efficacy of decitabine in combination with temozolomide in metastatic melanoma: a phase I/II study and pharmacokinetic analysis. Ann Oncol. 2013:24(4):1112-9.

39. Zakharia $Y$, Monga $V$, Swami U, Bossler AD, Freesmeier M, Frees $M$, et al. Targeting epigenetics for treatment of BRAF mutated metastatic melanoma with decitabine in combination with vemurafenib: a phase lb study. Oncotarget. 2017:8(51):89182-93.

40. Greenberg ES, Chong KK, Huynh KT, Tanaka R, Hoon DS. Epigenetic biomarkers in skin cancer. Cancer Lett. 2014:342(2):170-7.

41. Sun V, Zhou WB, Majid S, Kashani-Sabet M, Dar AA. MicroRNA-mediated regulation of melanoma. Br J Dermatol. 2014;171(2):234-41.

42. Karahoca M, Momparler RL. Pharmacokinetic and pharmacodynamic analysis of 5-aza-2'-deoxycytidine (decitabine) in the design of its dose-schedule for cancer therapy. Clin Epigenetics. 2013;5(1):3.

43. Pencheva N, Tran H, Buss C, Huh D, Drobnjak M, Busam K, et al. Convergent multi-miRNA targeting of ApoE drives LRP1/LRP8-dependent melanoma metastasis and angiogenesis. Cell. 2012;151(5):1068-82.

44. Yang X, Lei S, Long J, Liu X, Wu Q. MicroRNA-199a-5p inhibits tumor proliferation in melanoma by mediating HIF-1alpha. Mol Med Rep. 2016; 13(6):5241-7.

45. Cheung HH, Davis AJ, Lee TL, Pang AL, Nagrani S, Rennert OM, et al. Methylation of an intronic region regulates miR-199a in testicular tumor malignancy. Oncogene. 2011;30(31):3404-15.

46. Mudduluru G, Ceppi P, Kumarswamy R, Scagliotti GV, Papotti M, Allgayer H. Regulation of Axl receptor tyrosine kinase expression by miR-34a and miR199a/b in solid cancer. Oncogene. 2011;30(25):2888-99.

47. Minna E, Romeo P, De Cecco L, Dugo M, Cassinelli G, Pilotti S, et al. miR199a-3p displays tumor suppressor functions in papillary thyroid carcinoma. Oncotarget. 2014:5(9):2513-28.

48. Huang J, Dong B, Zhang J, Kong W, Chen Y, Xue W, et al. miR-199a-3p inhibits hepatocyte growth factor/c-met signaling in renal cancer carcinoma. Tumour Biol. 2014;35(6):5833-43.

49. Wu D, Huang HJ, He CN, Wang KY. MicroRNA-199a-3p regulates endometrial cancer cell proliferation by targeting mammalian target of rapamycin (mTOR). Int J Gynecol Cancer. 2013;23(7):1191-7.

50. Qu Y, Huang X, Li Z, Liu J, Wu J, Chen D, et al. miR-199a-3p inhibits aurora kinase $a$ and attenuates prostate cancer growth: new avenue for prostate cancer treatment. Am J Pathol. 2014;184(5):1541-9.
51. Marzese DM, Scolyer RA, Roque M, Vargas-Roig LM, Huynh JL, Wilmott JS, et al. DNA methylation and gene deletion analysis of brain metastases in melanoma patients identifies mutually exclusive molecular alterations. Neuro-Oncology. 2014;16(11):1499-509.

52. Tost J, Gut IG. DNA methylation analysis by pyrosequencing. Nat Protoc. 2007:2(9):2265-75.

53. Hochberg Y, Benjamini Y. More powerful procedures for multiple significance testing. Stat Med. 1990:9(7):811-8.
Ready to submit your research? Choose BMC and benefit from:

- fast, convenient online submission

- thorough peer review by experienced researchers in your field

- rapid publication on acceptance

- support for research data, including large and complex data types

- gold Open Access which fosters wider collaboration and increased citations

- maximum visibility for your research: over $100 \mathrm{M}$ website views per year

At $\mathrm{BMC}$, research is always in progress.

Learn more biomedcentral.com/submissions 